\title{
Highly Selective Needle-Type Glucose Sensors Prepared by the Immobilization of Glucose Oxidase on $\gamma$-Polyglutamic Acid Film
}

\author{
Mikito Yasuzawa, ${ }^{* \dagger}$ Kazuaki Edagawa, ${ }^{*}$ Takuya Matsunaga, ${ }^{*}$ Hiroki TaKaOKA, ${ }^{*}, * *$ and \\ Tomoki YABUTANI*
}

\author{
*Department of Chemical Science and Technology, Institute of Science and Technology, \\ The University of Tokushima, 2-1 Minamijosanjima, Tokushima 770-8506, Japan \\ **Toyo Precision Parts MFG. Co., Ltd., Kashihara, Nara 634-0836, Japan
}

\begin{abstract}
Fine needle-type glucose sensors with an outer diameter of less that $0.2 \mathrm{~mm}$ were fabricated using a low-cost biodegradable $\gamma$-polyglutamic acid (PGA) not only as a glucose oxidase (GOx) immobilizing material, but also as a permselective inner membrane. PGA film was prepared on a cellulose acetate-coated Pt-Ir wire electrode, and GOx was covalently immobilized on its surface using water-soluble carbodiimide. The obtained electrode was practically not affected by the existence of electroactive compounds, and provided long-term stability for approximately one month. It also functioned in horse serum with a good linear relationship between the current and glucose concentrations within the range of 2.8 to $22.4 \mathrm{mM}$.
\end{abstract}

(Received November 9, 2010; Accepted January 4, 2011; Published March 10, 2011)

\section{Introduction}

The number of diabetics has rapidly increased around the world, due to changes in life-style, diet and aging. It is well known that keeping good control of the blood glucose level can prevent the onset and progression of serious diabetes complications. Therefore, it is important to accurately recognize the blood glucose level in order to make appropriate treatments, such as insulin therapy. Although self monitoring of the blood glucose concentration with a finger prick is a generally used method, the development of implantable glucose sensors for a continuous glucose monitoring system (CGMS) has provided significant benefit, since patients with insulin-dependent (type 1) diabetes have fear of hypoglycemia, especially during sleep at night. ${ }^{1,2}$ Although several non-invasive CGMS, such as GlucoWatch using reverse iontophoresis, ${ }^{3}$ and OrSense NBM-200G using occlusion spectroscopy, ${ }^{4}$ have already been released, invasive-type CGMS using needle-type amperometric enzyme sensors, such as Medtronix MiniMed Guardian Continuous Monitoring System, Abbott Diabetes Care FreeStyle Navigator and DexCom STS-7, have become greatly popularized, since the accuracy to detect low blood glucose level is higher than that of non-invasive CGMS. ${ }^{1-3,5-8}$ However, efforts to minimize the invasivity of the CGMS are strongly demanded. In order to obtain a practical implantable glucose sensor, several items must be completed, such as steady immobilization of the enzyme for satisfactory sensor long-term stability, highly selective detection for accurate glucose measurements, sensor surface biocompatibility for successful measurements in a biological medium, and disinfective and non poisonous for in vivo usage safety. Among all of these subjects, usage safety is the first

† To whom correspondence should be addressed.

E-mail: mik@chem.tokushima-u.ac.jp priority for the development of sensors for in vivo measurements. Therefore, selection of the material for fabricating the sensor is of critical importance.

Evaluating the safety of medical products is achieved based on ISO10993 of the global standard by Ministry of Health, Labor and Welfare in Japan or Food and Drug Administration (FDA). The elimination of animal-origin materials is an adequate instruction, since the uses of such materials contain risks of unknown infections and prion diseases. ${ }^{9-12}$ In addition, the use of perfluorinated chemicals (PFCs) has attracted regulatory concern and/or action globally, since perfluorinated substances are known to be persistent. ${ }^{13}$ Some of them are bioaccumulating, particularly those with long carbon chains, and some have been reported to cause toxic effects in laboratory animals, for which the relevance to human health cannot be dismissed. ${ }^{14,15}$ The most known perfluorinated compound from everyday life is probably the perfluorinated polymer polytetrafluoroethylene (PTFE), which is used as a "non-stick" coating in kitchenware and laboratory equipment. PTFE has also been applied in medical fields, such as catheters, medical filters, and artificial blood vessels. In addition, PTFE-coated wire is one of the most employed electrode material for biosensor fabrication. ${ }^{16-19}$ Moreover, perfluorinated-sulfonated ion-exchange polymer "Nafion ${ }^{\circledR}$ " is another popularly used material in the fabrication of biosensors, since it is known to be effective for eliminating the influence of electroactive compounds on the sensor response by an electrostatic interaction. ${ }^{19-28}$

Recently, a low-cost non-animal origin biodegradable polyamino acid, $\gamma$-polyglutamic acid (PGA), has received attention for clinical applications due to its good biocompatibility. ${ }^{29}$ We previously reported that PGA can be employed as an enzyme immobilizing material for the preparation of a fine needle-type glucose sensor. ${ }^{28}$ The sensor properties of the obtained GOx-immobilized electrode were comparable to that of a sensor prepared by the most commonly used method, cross-linking of 


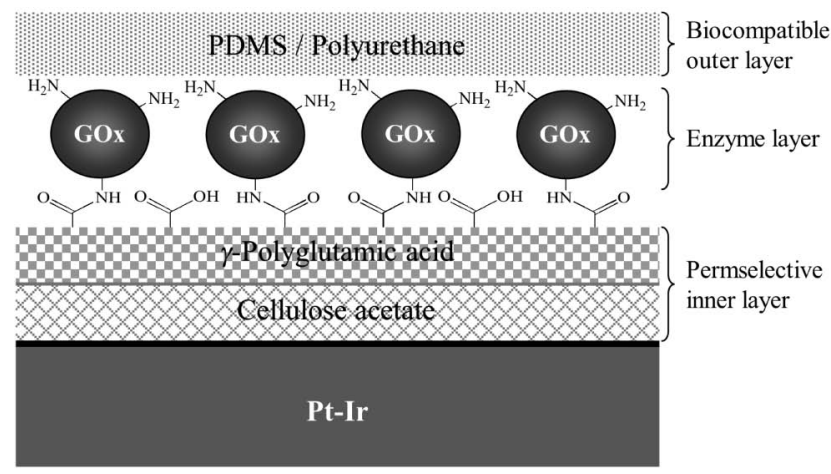

Fig. 1 Schematic illustration of the sensing region of the glucose sensor.

GOx and bovine serum albumin with glutardehyde. ${ }^{20}$ However, Nafion, together with cellulose acetate, was employed as an inner film in order to eliminate the influence of electroactive compounds existing in biological fluids, such as ascorbic acid, uric acid and acetaminophen. Considering that the utmost employment of low environmental load materials will be a higher requirement from now on, it is advisable that the use of PFCs should be reduced by possible means. Since PGA is a polyamide with numerous carboxyl groups on the side chain, it is acceptable that PGA will perform as a negatively charged permselective film equivalent to Nafion.

In this study, PGA was employed not only as a substrate for enzyme immobilization, but also as a permselective film, which will eliminate the influence of electroactive compounds in combination with cellulose acetate. The glucose sensors consisted of three layers: a permselective inner layer, an enzyme layer, and a biocompatible outer layer (Fig. 1). The enzyme layer was prepared by the covalent attachment of GOx on PGA film with amide bonds, generated by the reaction of a lysine residue of GOx and carboxyl group of PGA in the presence of a water-soluble condensation agent, 1-ethyl-3-(3-dimethylaminopropyl) carbodiimide hydrochloride (EDC). The properties of the obtained sensors were examined in a phosphate buffer solution ( $\mathrm{pH} \mathrm{7.4)}$ and horse serum at $40^{\circ} \mathrm{C}$, and the utilities of low environmental load material PGA as an enzyme-immobilizing material and permselective film were evaluated. In addition, a GOx-immobilized electrode with Nafion inner film was prepared for comparing the sensor properties.

\section{Experimental}

\section{Reagents and chemicals}

Glucose oxidase (GOx) (265 U/mg, purified from Aspergillus niger) was purchased from Biozyme Laboratories. $\gamma$-Polyglutamic acid $\left(M_{\mathrm{w}}=800000-1000000\right)$ was kindly supplied from Nippon Poly-Glu. A water-soluble condensing agent, 1-ethyl-3(3-dimethylaminopropyl) carbodiimide hydrochloride (EDC), was obtained from Toyo Kasei Kogyo. Cellulose acetate was obtained from Kishida Chemical. Polyurethane (Tecoflex) was purchased from Thermedics Inc. (Woburn, MA). Polydimethylsiloxane (MED-4211) was purchased from Nusil (Carpenteria, CA). Nafion ${ }^{\circledR}$ (perfluoronated ion-exchange powder $5 \%$ wt. in a mixture of lower aliphatic alcohols and water) was purchased from Aldrich. Horse serum containing $0.55 \mathrm{mmol} \mathrm{dm}^{-3}$ glucose was purchased from Tissue Culture Biologicals (Los Alamitos, CA). Platinum-iridium (0.10 $\mathrm{mm}$ in diameter, Pt $90 \%-\mathrm{Ir} 10 \%)$ wire was purchased from Nilaco. The insulation tube of polyimide $(0.12 \mathrm{~mm}$ i.d., $0.16 \mathrm{~mm}$ o.d.) was purchased from Furukawa Electric. All other reagents were of analytical grade, and were used without further purification.

\section{Preparation of a fine needle-type glucose sensor}

Pt-Ir wire (2.0 mm length of $30 \mathrm{~mm}$ length) was used as a sensing region. The sensor fabrication consisted of three main steps: permselective inner-film preparation, enzyme immobilization and biocompatible outer-film construction. The inner film was prepared by alternate five-times coatings of cellulose acetate (CA) using a 5\% cellulose acetate solution, following five-times coatings of PGA using a 5\% PGA dimethyl sulfoxide (DMSO) solution. Immobilization of the enzyme was performed by pouring a mixture solution of $10 \mathrm{mg} \mathrm{mL}^{-1} \mathrm{GOx}$ and $0.125 \%$ (v/v) EDC on a PGA coated surface, and dried for $30 \mathrm{~min}$ at room temperature. After the construction of an outer film prepared using a tetrahydrofuran solution containing $4.3 \mathrm{wt} \%$ polyurethane (PU) and $1.3 \mathrm{wt} \%$ polydimethylsiloxane (PDMS), the electrode was dried at room temperature for 3 days and immersed in $0.1 \mathrm{~mol} \mathrm{dm}^{-3}$ PBS for at least 2 days before use. The outer diameter of the thickest part of the sensors was less than $0.2 \mathrm{~mm}$. The obtained electrode is referred to herein as "GOx/PGA-CA". The preparation of a GOx-immobilized electrode with Nafion inner film was carried out according to methods of Wilson et al. ${ }^{19,20}$ That is, firstly, inner films were prepared by alternate five-times coatings of both Nafion and cellulose acetate using a 5\% Nafion solution and a 5\% cellulose acetate solution, respectively. Secondly, a mixture solution of bovine serum albumin, GOx, and glutaldehyde was poured on the inner film-coated electrode surface. Finally, an outer film was prepared on GOx-immobilized film using the same method performed on the fabrication of GOx/PGA-CA. The electrode obtained using Nafion inner film is referred to herein as "GOx/Nafion-CA".

\section{Sensor measurement procedure}

The principle for determining the current response is based on the formation of hydrogen peroxide during the enzyme catalytic reaction.

The amperometric responses of the prepared electrodes to glucose were examined at $40^{\circ} \mathrm{C}$ in a $0.1 \mathrm{M}$ phosphate buffer solution of $\mathrm{pH} 7.4$ containing $0.1 \mathrm{~mol} \mathrm{dm}^{-3} \mathrm{NaCl}$ by measuring the electrooxidation current at a potential of $0.6 \mathrm{~V}(v s . \mathrm{Ag} / \mathrm{AgCl})$ for hydrogen peroxide detection. Amperometric measurements were performed with a Potentiostat Model 3104 (Pinnacle Technology Inc.).

The background current was stabilized with in $15 \mathrm{~min}$. Calibration of the sensor was carried out by adding increasing amounts of glucose to the stirred buffer solution. The current was measured at the plateau (steady-state response), and was related to the concentration of the analyte. The steady-state response currents were obtained within $60 \mathrm{~s}$ for all electrodes. The sensor response was also measured in horse serum containing $0.55 \mathrm{mM}$ glucose at $40^{\circ} \mathrm{C}$ in order to evaluate the performance of the sensor in biological media.

\section{Results and Discussion}

\section{Sensor response in phosphate buffer saline}

Figure 2 shows typical calibration curves of GOx/PGA-CA and GOx/Nafion-CA for a comparison. The response current increased with increasing concentration of glucose up to $22.4 \mathrm{mM}$, while the linear relationship between the glucose 


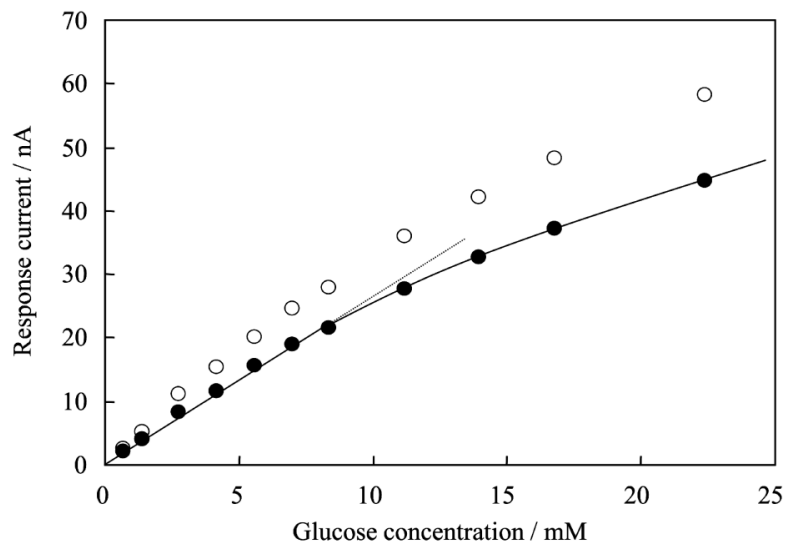

Fig. 2 Typical calibration curves of GOx/PGA-CA (closed circles) and GOx/Nafion-CA (open circles), measured in a $0.1 \mathrm{M}$ phosphate buffer solution $(\mathrm{pH} 7.4)$ at $40^{\circ} \mathrm{C}$.

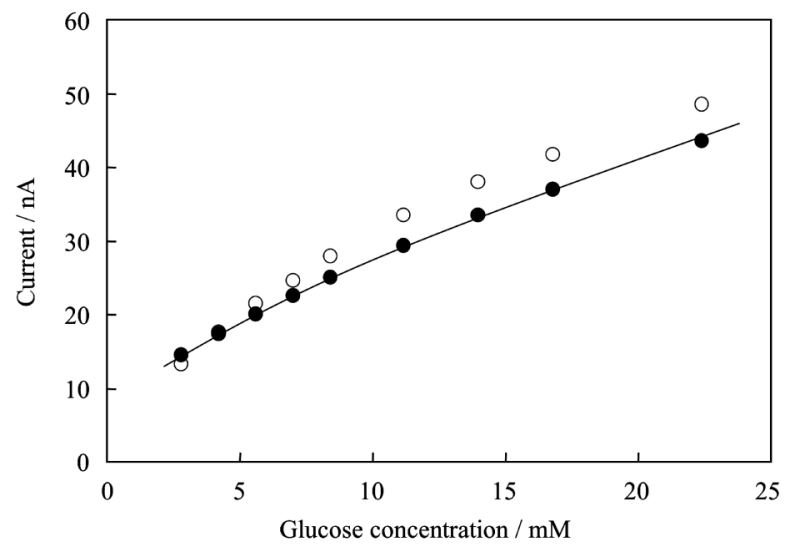

Fig. 3 Variation of current with the glucose concentration for GOx/PGA-CA (closed circles) and GOx/Nafion-CA (open circles) measured in horse serum containing $0.55 \mathrm{mM}$ glucose at $40^{\circ} \mathrm{C}$.

concentration and the response current was obtained within a range lower than $8.4 \mathrm{mM}$ for both electrodes (correlation coefficient, $\left.r^{2}>0.98\right)$. Obviously, a sensor without Nafion inner film was shown to work as well as that with Nafion inner film. Considering the application of the sensor to in vivo measurements, it is desired to monitor the physiological glucose concentrations in the $2-25 \mathrm{mM}$ range. Therefore, further achievement for extending the linear response range, such as optimization of the diffusion-limiting outer membrane thickness, is required.

\section{Sensor response in horse serum}

The variation of the current with the glucose concentration in horse serum containing $0.55 \mathrm{mM}$ glucose at $40^{\circ} \mathrm{C}$, for GOx/PGA-CA (closed circles) and GOx/Nafion-CA (open circles), is illustrated in Fig. 3. Obviously, the sensor without Nafion inner film was shown to also work well in horse serum. The response current of both electrodes increased with increasing concentration of glucose up to $22.4 \mathrm{mM}$. Nevertheless, the response to glucose was determined to be approximately $25 \%$ lower in horse serum compared with that in PBS from the ratio of the slope calculated within the range of 2.8 and $8.4 \mathrm{mM}$. A lower current observed in horse serum arose from the adsorption of protein on the surface of the electrode, which lowered the
Table 1 Influence of interferents on the glucose response current in a $0.1 \mathrm{M}$ phosphate buffer solution ( $\mathrm{pH}$ 7.4) containing $0.1 \mathrm{M} \mathrm{NaCl}$

\begin{tabular}{lccc}
\hline \multirow{2}{*}{ Electrode } & \multicolumn{3}{c}{$i_{\mathrm{G}+\mathrm{i}} / i_{\mathrm{G}}{ }^{\mathrm{a}}$} \\
\cline { 2 - 4 } & Ascorbic acid $^{\mathrm{b}}$ & Uric acid $^{\mathrm{b}}$ & Acetaminophen $^{\mathrm{b}}$ \\
\hline GOx/PGA-CA & 1.00 & 1.01 & 1.05 \\
GOx/Nafion-CA & 1.00 & 1.00 & 1.02 \\
\hline
\end{tabular}

a. $i_{\mathrm{G}}$, Response current of glucose $(5.6 \mathrm{mM}) ; i_{\mathrm{G}+1}$, response current of glucose $(5.6 \mathrm{mM})$ in the presence of interferent at physiological maximum.

b. Physiological maximum concentration: ascorbic acid, $0.11 \mathrm{mM}$; uric acid, $0.48 \mathrm{mM}$; acetaminophen, $0.17 \mathrm{mM}$.

diffusion of glucose into the enzyme layer. ${ }^{30}$ However, the correlation coefficient between the glucose concentration and the response current obtained within the range of $2.8-22.4 \mathrm{mM}$ was higher than 0.98 . A wider linear relationship range obtained in horse serum compared with that in buffer solution might be due to a diffusion-limiting effect of protein film formed on the surface of the electrode.

\section{Long-term stability}

The variation of the response current in time on GOx/PGA-CA was tested in $5.6 \mathrm{mM}$ glucose at different intervals at $40^{\circ} \mathrm{C}$ and stored in phosphate buffer at $4^{\circ} \mathrm{C}$ when not in use. The electrode provided a stable response for more than about one month, while a decrease in the response was observed on the first two days. The initial decrease of response may have been due to the release of a weakly attached enzyme from the electrode surface. No clear distinction or damage was observed, even after one month of use.

\section{Influence of electoroactive compounds}

The interferences of electroactive compounds (interferents) existing in biological fluids to the glucose response were examined in the presence of their physiological maximum levels with the glucose concentration at $5.6 \mathrm{mM}$ (Table 1). The level of interference is expressed in Table 1 as $i_{\mathrm{G}+1} / i_{\mathrm{G}}$ : the ratio of the response current of glucose to the response current of glucose in the presence of an interferent. GOx/PGA-CA was approximately not affected by the existence of interferents, such as ascorbic acid, uric acid and acetaminophen. This indicated that PGA film, which was employed for the immobilization of enzyme, also functioned as an effective permselective film in order to eliminate interferents in combination with cellulose acetate.

\section{Conclusions}

This study demonstrates that a low-cost and non-animal origin polyamide, $\gamma$-polyglutamic acid (PGA) is a useful material for preparing a glucose sensor. The obtained glucose sensor was not influenced by the existence of electroactive compounds, such as ascorbic and uric acids, and acetaminophen, and presented a stable unchanged sensor response for one month, measured in a phosphate buffer solution of $\mathrm{pH} 7.4$ at $40^{\circ} \mathrm{C}$. This indicated that PGA functioned as a sufficient permselective film material, in addition as an enzyme immobilizing material. Although an extension of the linear relationship range was desirable for practical use, the sensor showed good sensor response even in horse serum. 


\section{Acknowledgements}

The authors are grateful to Nippon Poly-Glu Co. Ltd., Japan for providing $\gamma$-polyglutamic acids.

\section{References}

1. Juvenile Diabetes Research Foundation Continuous Glucose Monitoring Study Group, Diabetes Care, 2010, 33, 1004.

2. A. P. Cemeroglu, R. Stone, L. Kleis, M. S. Racine, D. C. Postellon, and M. A. Wood, Pediatr. Diabetes, 2010, 11, 182.

3. The Diabetes Research in Children Network (DirecNet) Study Group, Diabetes Care, 2004, 27, 722.

4. O. Amir, D. Weinstein, S. Zilberman, M. Less, D. Perl-Treves, H. Primack, A. Weinstein, E. Gabis, B. Fikhte, and A. Karasik, J. Diabetes Sci. Technol., 2007, 1, 463.

5. B. Kovatchev, S. Anderson, L. Heinemann, and W. Clarke, Diabetes Care, 2008, 31, 1160.

6. B. Bode, K. Gross, N. Rikalo, S. Schwartz, T. Wahl, C. Page, T. Gross, and J. Mastrototaro, Diabetes Technol. Ther., 2004, 6, 105.

7. R. L. Weinstein, S. L. Schwartz, R. L. Brazg, J. R. Bugler, T. A. Peyser, and G. V. McGarraugh, Diabetes Care, 2007, 30, 1125 .

8. S. K. Garg, J. Smith, C. Beatson, B. Lopez-Baca, M. Voelmle, and P. A. Gottlieb, Diabetes Technol. Ther., 2009, 11,65 .

9. C. N. Berger, P. L. Donne, and H. Windemann, Biologicals, 2005, 33, 1 .

10. Evaluation of medical devices incorporating products of animal origin, Compilation prepared by European Commission (EC), http://ec.europa.eu/enterprise/medical_ devices/meddev/2_5-8_02-1999.pdf/.

11. Application of Council Directive 93/42/EEC taking into account the Commission Directive 2003/32/EC for Medical Devices utilising tissues or derivatives originating from animals for which a TSE risk is suspected, Compilation prepared by European Commission (EC), http://ec.europa. eu/enterprise/medical_devices/meddev/2_11_1_rev2_bsetse_ january2008.pdf/.

12. A Practical Guide to ISO 10993: Part 1-Introduction to the Standards, Compilation prepared by Medical Device Link, http://www.devicelink.com/mddi/archive/98/01/023. $\mathrm{html} /$.

13. P. Rostkowski, S. Taniyasu, Y. Miyake, K. Y. Kwok, L. W. Y. Yeung, P. Lam, K. Kannan, and N. Yamashita, Organohal. Comp., 2007, 69, 2812.

14. Environmental Protection Agency, Federal Register, 2010, $75,4292$.

15. S. D. Richardson, Anal. Chem., 2010, 82, 4742.

16. M. Bugli, D. Moscone, P. Rodino, and G. Palleschi, Clin. Chem. Enzymol. Commun., 1995, 7, 17.

17. B. Linke, W. Kerner, M. Kiwit, M. Pishko, and A. Heller, Biosens. Bioelectron., 1994, 9, 151.

18. P. Dungel, N. Long, B. Yu, Y. Moussy, and F. Moussy, J. Biomed. Mater. Res., 2009, 85, 699.

19. R. Gifford, M. M. Batchelor, Y. Lee, G. Gokulrangan, M. E. Meyerhoff, and G. S. Wilson, J. Biomed. Mater. Res., 2005, 75, 755 .

20. D. S. Bindra and G. S. Wilson, Anal. Chem., 1989, 61, 2566.

21. F. Mizutani, S. Yabuki, and T. Katsura, Anal. Chim. Acta, 1993, 274, 201.

22. F. Moussy, S. Jakeway, J. Harrison, and R. V. Rajotte, Anal. Chem., 1994, 66, 3882.

23. Y. Zhang, Y. Hu, G. S. Wilson, D. Moatti-Sirat, V. Poitout, and G. Reach, Anal. Chem., 1994, 66, 1183.

24. I. L. Mattos, L. V. Lukachova, L. Gorton, T. Laurell, and A. A. Karyakin, Talanta, 2001, 54, 963.

25. J.-J. Xu, Z.-H. Yu, and H.-Y. Chen, Anal. Chim. Acta, 2002, $463,239$.

26. M. Yasuzawa and A. Kunugi, Electrochem. Commun., 1999, $1,459$.

27. M. Yasuzawa, M. Kitahara, T. Yabutani, and J. Motonaka, Bunseki Kagaku, 2009, 58, 941.

28. H. Takaoka and M. Yasuzawa, Anal. Sci., 2010, 26, 551.

29. W.-C. Lin, D.-G. Yu, and M.-C. Yang, Colloids Surf., B, 2006, 47,43 .

30. M. Jablecki and D. A. Gough, Anal. Chem., 2000, 72, 1853. 